\title{
Game Analysis of the Strategies for the Pre-sale Prices of New Products
}

\author{
Yanwei Shi ${ }^{1}$, Chunhui $\mathrm{Ma}^{2}$ \\ ${ }^{1}$ School of Intelligent Science and Information Engineering in Xi'an Peihua University, Xi'an, China 710125 \\ ${ }^{2}$ School of Science in Xi'an University of Architecture and Technology, Xi'an, China 710055
}

Keywords: duopoly market; pre-sale price; equilibrium; backward induction

\begin{abstract}
The main purpose of this paper is to study the strategies for the pre-sale prices of new products chosen by firms in game theory. Firstly, for the differentiated new products, the equilibrium of the strategies for the pre-sale prices of new products chosen by firms at the same time is analyzed in duopoly market. Then, the backward induction solution is discussed when the pre-sale prices of new products are chosen by firms at the different times. Finally, by comparing the profits in two cases, it is obtained that the firm that first chosen the pre-sale price of new product will get more profits.
\end{abstract}

\section{Introduction}

With the continuous development of economy and the further deepening of market, while strengthening the production and sales of products, firms gradually increase their investment in research and development, production and sales of new products, in order to make firms have better vitality and competitiveness. In the sales process of new products, the pre-sale strategy is one of the strategies adopted by firms in recent years.

The pre-sale strategy is one of new product sales strategies that firms begin to accept consumer orders before the formal sale or normal sales season of new products. In increasingly developed information and network society, the pre-sale strategy has been more common. For example, when new fashions, new mobile phones and new cars are introduced, firms often hold a new product meeting. At the conference, firm will show the advantages of new product such as new style, new functions, new features, and the pre-sale prices of new products, and accept consumers' orders online and offline. The firm adopts the pre-sale strategy when introducing new products. On the one hand, firm can obtain sales funds in advance, update the demand forecast information of new products according to the pre-sale quantity, so as to reduce the inventory risk. On the other hand, firm can also combine the pre-sale strategy of new products with the strategies of pricing, information disclosure, price guarantee and innovation of new products, in order to further increase the profits of firms.

Duopoly model is a common market model in economic theory. In free market, if the market demand of a certain product is controlled by two firms, this phenomenon is called duopoly. The model used to describe the phenomenon of duopoly is called duopoly model [1-5]. In 1838, French economist Cournot first studied this market structure and established the Cournot duopoly model. Later, von Neumann and Oscar Morgenstern discussed the equilibrium in this model. The discussion of Nash equilibrium has been one of the topics in economics and game theory [6-8].

In this paper, the equilibrium of the strategies for the pre-sale prices of new products chosen by firms is studied in duopoly market. Firstly, for two firms producing the differentiated products, the equilibrium of the strategies for the pre-sale prices of new products chosen by firms at the same time is analyzed in duopoly market. Then, the solution of backward induction is discussed in the case of the pre-sale prices of new products chosen by firms at the different times. Finally, by comparing the profits in two cases, it is obtained that the firm that first chosen the pre-sale price of new product will get more profit.

\section{The pre-sale prices chosen at the same time}

In a free market, it is assumed that only two firms compete, and some products in the market are 
produced and sold only by the firms. In the competition, the firms choose the pre-sale prices of new products. We only consider a kind of differentiated new products. The two firms are denoted as firm 1 and firm 2, respectively. The pre-sale prices of new product, which are chosen by firm 1 and firm 2, are $p_{1}$ and $p_{2}$, respectively. And the demands of consumers for the new product of firm 1 and firm 2, i.e. the outputs of firm 1 and firm 2, are $q_{1}=a-p_{1}+b p_{2}$ and $q_{2}=a-p_{2}+b p_{1}$, where $b>0$ is the substitution coefficient between firm 1's new product and firm 2's new product. We also assume that there are no fixed costs in the productions of firm 1 and firm 2, and marginal costs are both $c$ $(c<a)$.

In this section, we consider the case that firm 1 and firm 2 choose the pre-sale prices of new product at the same time. We assume that there is no meaning if the pre-sale price of new product is less than 0 , and the pre-sale price of new product is nonnegative. So, the profits of firm 1 and firm 2 are $\pi_{1}=q_{1}\left(p_{1}-c\right)=\left(a-p_{1}+b p_{2}\right)\left(p_{1}-c\right)$ and $\pi_{2}=q_{2}\left(p_{2}-c\right)=\left(a-p_{2}+b p_{1}\right)\left(p_{2}-c\right)$, respectively.

Given the combination of the pre-sale prices of new product $\left(p_{1}^{*}, p_{2}^{*}\right)$ be the equilibrium in this case. Then, for firm 1 and firm 2, $p_{1}^{*}$ and $p_{2}^{*}$ are the solutions of the following optimization problems:

$$
\max _{0 \leq p_{1}<+\infty} \pi_{1}=\max _{0 \leq p_{1}<+\infty}\left(a-p_{1}+b p_{2}^{*}\right)\left(p_{1}-c\right) \text { and } \max _{0 \leq p_{2}<+\infty} \pi_{2}=\max _{0 \leq p_{2}<+\infty}\left(a-p_{2}+b p_{1}^{*}\right)\left(p_{2}-c\right) .
$$

Solve this optimization problems for firm 1 and firm 2, $p_{1}^{*}=\frac{1}{2}\left(a+b p_{2}^{*}+c\right)$ and $p_{2}^{*}=\frac{1}{2}\left(a+b p_{1}^{*}+c\right)$. Therefore, $p_{1}^{*}=p_{2}^{*}=\frac{a+c}{2-b}$.

Thus, in the case that firm 1 and firm 2 choose the pre-sale prices of new product at the same time, the equilibrium pre-sale prices of new product are both $p^{*}=p_{1}^{*}=p_{2}^{*}=\frac{a+c}{2-b}$. Now, the equilibrium outputs of firm 1 and firm 2 are $q^{*}=q_{1}{ }^{*}=q_{2}{ }^{*}=\frac{a+c}{2-b}-c$, and the total equilibrium output in the market is $Q^{*}=q_{1}{ }^{*}+q_{2}{ }^{*}=\frac{2 a+b c}{2-b}-c$. Therefore, the equilibrium profits of firm 1 and firm 2 are both $\pi^{*}=\pi_{1}^{*}=\pi_{2}^{*}=\left(\frac{a+c}{2-b}-c\right)^{2}$, and the total equilibrium profit in the market is $\Pi^{*}=\pi_{1}^{*}+\pi_{2}^{*}=2\left(\frac{a+c}{2-b}-c\right)^{2}$.

\section{The pre-sale prices chosen at the different times}

In this section, we will consider the case that firm 1 and firm 2 choose the pre-sale prices of new product at the different times. We assume that the firm that first chooses the pre-sale price of new product is firm 1, and the firm that later chooses the pre-sale price of new product is firm 2 . The time-orders in this case are as follows:

STAGE 1 . Firm 1 first chooses the pre-sale price of its new product $p_{1} \geq 0$;

STAGE 2. After observing firm 1's pre-sale price of new product $p_{1}$, firm 2 chooses the pre-sale price of its new product $p_{2} \geq 0$;

STAGE 3. The profits of firm 1 and firm 2 are $\pi_{1}=\left(a-p_{1}+b p_{2}\right)\left(p_{1}-c\right)$ and $\pi_{2}=\left(a-p_{2}+b p_{1}\right)\left(p_{2}-c\right)$, respectively.

Let $R_{2}\left(p_{1}\right)$ be the optimal response of firm 2 to firm 1's pre-sale price of new product $p_{1}$. Then $R_{2}\left(p_{1}\right)$ should satisfy $\max _{p_{2} \geq 0} \pi_{2}=\max _{p_{2} \geq 0}\left(a-p_{2}+b p_{1}\right)\left(p_{2}-c\right)$. So, $R_{2}\left(p_{1}\right)=\frac{1}{2}\left(a+b p_{1}+c\right)$. 
In the previous section, we have analyzed the case that the pre-sale prices of new product chosen by firm 1 and firm 2 at the same time. The optimal responses of firm 2 are same both in this section and in the previous section. The difference between in two cases is that $R_{2}\left(p_{1}\right)$, in this section, is the optimal responses of firm 2 after observing firm 1's pre-sale price of new product, but in the previous section, it is the optimal responses of firm 2 to assumed any firm 1's pre-sale price of new product, and the pre-sale prices of new product chosen by firm 1 and firm 2 are at the same time.

Since firm 1 can also predict the optimal response $R_{2}\left(p_{1}\right)$ of firm 2 as firm 2 does, firm 1 can predict that, when the pre-sale price of its new product is $p_{1}$, firm 2 will choose the pre-sale price of new product by the optimal response $R_{2}\left(p_{1}\right)$. Then, in stage 1 , firm 1 's choice should satisfy

$$
\max _{p_{1} \geq 0} \pi_{1}=\max _{p_{1} \geq 0}\left(a-p_{1}+b R_{2}\left(p_{1}\right)\right)\left(p_{1}-c\right)=\max _{p_{1} \geq 0}\left(a-p_{1}+\frac{1}{2} b\left(a+b p_{1}+c\right)\right)\left(p_{1}-c\right) .
$$

Thus,

$$
p_{1}=\frac{(a+c)(2+b)-b^{2} c}{4-2 b^{2}} \text { and } p_{2}=R_{2}\left(p_{1}\right)=\frac{(a+c)\left(4-b^{2}+2 b\right)-b^{3} c}{8-4 b^{2}} .
$$

This is the solution of backward induction, i.e. in the case of the pre-sale prices of new product chosen by firm 1 and firm 2 at the different times, the solution of backward induction

$$
\left(p_{1}, p_{2}\right)=\left(p_{1}, R_{2}\left(p_{1}\right)\right)=\left(\frac{(a+c)(2+b)-b^{2} c}{4-2 b^{2}}, \frac{(a+c)\left(4-b^{2}+2 b\right)-b^{3} c}{8-4 b^{2}}\right) \text {. }
$$

Hence, the backward induction pre-sale prices of new product chosen by firm 1 and firm 2 are $p_{1}=\frac{(a+c)(2+b)-b^{2} c}{4-2 b^{2}}$ and $p_{2}=\frac{(a+c)\left(4-b^{2}+2 b\right)-b^{3} c}{8-4 b^{2}}$, respectively. Now, the backward induction outputs of firm 1 and firm 2 are

$$
q_{1}=a-\frac{(a+c)(2+b)-b^{2} c}{4-2 b^{2}}+\frac{b(a+c)\left(4-b^{2}+2 b\right)-b^{4} c}{8-4 b^{2}}
$$

and

$$
q_{2}=a-\frac{(a+c)\left(4-b^{2}+2 b\right)-b^{3} c}{8-4 b^{2}}+\frac{b(a+c)(2+b)-b^{3} c}{4-2 b^{2}} .
$$

And the total backward induction output in the market is

$$
Q=q_{1}+q_{2}=2 a+\frac{\left(b^{2} c-(a+c)(2+b)\right)(1-b)}{4-2 b^{2}}+\frac{\left(b^{3} c-(a+c)\left(4-b^{2}+2 b\right)\right)(1-b)}{8-4 b^{2}} .
$$

Therefore, the backward induction profits of firm 1 and firm 2 are

$$
\pi_{1}=\left[a-\frac{(a+c)(2+b)-b^{2} c}{4-2 b^{2}}+b \frac{(a+c)\left(4-b^{2}+2 b\right)-b^{3} c}{8-4 b^{2}}\right]\left[\frac{(a+c)(2+b)-b^{2} c}{4-2 b^{2}}-c\right],
$$

and

$$
\pi_{2}=\left[a-\frac{(a+c)\left(4-b^{2}+2 b\right)-b^{3} c}{8-4 b^{2}}+b \frac{(a+c)(2+b)-b^{2} c}{4-2 b^{2}}\right]\left[\frac{(a+c)\left(4-b^{2}+2 b\right)-b^{3} c}{8-4 b^{2}}-c\right] .
$$

By contrast, we find $\pi_{1}>\pi^{*}>\pi_{2}$, that is, in the case of that firm 1 and firm 2 choose pre-sale prices of new products at the different times. Firm that first choose pre-sale price of new product will earn more profits than the later firm. And the profits are more than equilibrium profits in section 2 . At the same time, once pre-sale price of new product is chosen later, firms will lose more profits. 


\section{Conclusions}

Recall from Section 2 that the equilibrium pre-sale price of new product chosen by each firm is $p^{*}=p_{1}^{*}=p_{2}^{*}=\frac{a+c}{2-b}$. Thus, the backward induction pre-sale price of new product in Section 3 , $p_{1}=\frac{(a+c)(2+b)-b^{2} c}{4-2 b^{2}}$, is greater than the equilibrium pre-sale price of new product, $p^{*}=\frac{a+c}{2-b}$, so the output is lower in Section 2. In Section 3, however, firm 1 could have chosen its the equilibrium pre-sale price of new product, $p^{*}=\frac{a+c}{2-b}$, in which case firm 2 would have responded with its the equilibrium pre-sale price of new product. Thus, in Section 3, firm 1 could have achieved its equilibrium profit level but chose to do otherwise, so firm 1's profit in Section 3 must exceed its profit in Section 2. But the output is lower in Section 3, so profits are lower. Hence the fact that firm 1 is better off implies that firm 2 is worse off in Section 3 than in Section 2.

The observation that firm 2 does worse in the case of the pre-salt prices chosen at the same time than in the case of the pre-salt prices chosen at the different times illustrates an important difference between one firm and two firms decision problems. In one firm decision, having more information can never make the decision maker worse off. However, having more information, or more precisely, having it known to the other firms that one has more information) can make a firm worse off.

In the case of the pre-salt prices chosen at the different times, the information in question is the pre-sale price of new product chosen by firm 1: firm 2 knows $p_{1}$, and firm 1 knows that firm 2 knows $p_{1}$. To see the effect this information, consider the modified in the case of the pre-salt prices chosen at the different times, in which firm 1 chooses $p_{1}$, after which firm 2 chooses $p_{2}$ but does so without observing $p_{1}$. If firm 2 believes that firm 1 has chosen its backward induction pre-sale price of new product $p_{1}=\frac{(a+c)(2+b)-b^{2} c}{4-2 b^{2}}$, then firm 2's best response is again $R_{2}\left(p_{1}\right)=\frac{(a+c)\left(4-b^{2}+2 b\right)-b^{3} c}{8-4 b^{2}}$. But if firm 1 anticipates that firm 2 will hold this belief and so choose this pre-sale price of new product, then firm 1 prefers to choose its best response to $R_{2}\left(p_{1}\right)=\frac{(a+c)\left(4-b^{2}+2 b\right)-b^{3} c}{8-4 b^{2}}$ rather than its backward induction pre-sale price of new product $p_{1}$. Thus, firm 2 should not believe that firm 1 has chosen its backward induction pre-sale price of new product. Rather, the unique equilibrium of this modified in the case of the pre-salt prices chosen at the different times is for both firms to choose the equilibrium pre-sale price of new product $p^{*}=\frac{a+c}{2-b}$ in the case of the pre-salt prices chosen at the same time, where the firms move simultaneously. Thus, having firm 1 know that firm 2 knows $p_{1}$, firm 2 would loose.

\section{Acknowledgments}

This paper supported by transverse scientific research project of Xi'an Peihua university (Grant No. 2018103001) and basic research fund of Xi'an university of architecture and technology (Grant No. JC1709).

\section{References}

[1] Robert Gibbons. A Primer in Game Theory, Information and Control, Prentice Hall, 1994.

[2] Drew Fudenberg and Jean Tirole. Game Theory, MIT Press, 1991. 
[3] Weiying Zhang. Game and Society, Peking University press, 2013.

[4] Harold W. K.. Classics in Game theory, Princeton. NJ: Princeton University Pres, 1997.

[5] Myerson, R.. Game Theory: Analysis of Conflict, Cambridge and London: Harvard University Press, 1991.

[6] Osborne, M. and A. Rubinstein. A Course in Game Theory, Cambridge and London: The MIT Press, 1994.

[7] Shanshan Yang. A Study on Bidding Strategy of Power Generation Firms in China based on Game Theory, Journal of Harbin University of Science and Technology, vol. 22(3), 14-18, 2014.

[8] Fulin Li. The Game Between Popular Culture and Elite Culture from the Field Perspective -- a Reflection on Contemporary Chinese Aesthetic Culture, Journal of Shandong Normal University, vol. 17(2), 106-115, 2014. 